\title{
Câncer de Mama e Atividade Física: Percepções durante a Pandemia de Covid-19
}

doi: https://doi.org/10.32635/2176-9745.RBC.2021v67n2.1291

\author{
Breast Cancer and Physical Activity: Perceptions during the COVID-19 Pandemic \\ Cáncer de Mama y Actividad Física: Percepciones durante la Pandemia de Covid-19
}

Vanessa Bellani Lyra'; Tatiana de Bem Fretta²; Francine Stein³; Fabiana Flores Sperandio4; Adriana Coutinho de Azevedo Guimarães ${ }^{5}$

\section{RESUMO}

Introduçáo: $\mathrm{O}$ cenário de isolamento social imposto pela pandemia da Covid-19 tem representado um desafio para a saúde física e mental de mulheres em tratamento do câncer de mama. Objetivo: Investigar a percepção dos impactos da prática da atividade física remota nos sintomas clínicos e físicos de mulheres com câncer de mama durante o isolamento social imposto pela pandemia da Covid-19. Método: Estudo qualitativo e exploratório com 32 participantes com câncer de mama de um programa de extensão de uma universidade pública do Sul do Brasil. O roteiro de entrevista, aplicado nos meses de abril e maio de 2020, foi composto por 30 questóes abertas e fechadas, cujos dados foram transcritos na íntegra e analisados a partir da técnica de Análise de Discurso. Resultados: Três aspectos ganharam destaque na análise (categorias a priori), considerando-se o efeito do tempo, nomeadamente: a) aspectos pessoais e clínicos; b) aspectos físicos e clínicos antes e durante o isolamento social (nível de atividade física, dor e disposição geral); c) aspectos relacionados à prática de atividade física realizada de forma remota (percepçóes gerais acerca das adaptaçôes às atividades remotas e dificuldades em realizar a prática no ambiente domiciliar). Conclusáo: $\mathrm{O}$ isolamento social imposto pelo perigo iminente do contágio parece ter contribuído para o acirramento dos sintomas clínicos e físicos do câncer de mama, tais como o aumento percebido do linfedema e as dores, sobretudo no braço correspondente à cirurgia oncológica, além de refletir em uma diminuiçáo significativa da prática regular de atividade física. Palavras-chave: Exercício Físico; Neoplasias da Mama; Infecçóes por Coronavírus; Isolamento Social.

\section{ABSTRACT}

Introduction: The social isolation scenario of the COVID-19 pandemic is challenging for the physical and mental health of women in breast cancer treatment. Objective: To investigate the perception of the impacts of the practice of remote physical activity on the clinical and physical symptoms of women with breast cancer during the COVID-19 pandemic imposed social isolation. Method: Qualitative and exploratory study with 32 participants with breast cancer of a South public university extension program in Brazil. The interview script applied from April to May 2020 consisted of 30 open and closed questions, fully transcribed and analyzed based in the Discourse Analysis method. Results: Three aspects stood out in the analysis (a priori categories), considering the effect of the time, respectively: a) physical and clinical aspects; b) physical and clinical aspects before and during social isolation (level of physical activity, pain and general disposition); c) aspects related to physical activity practice carried out remotely (overall perceptions about adaptation to remote activities and difficulties while performing in home environment). Conclusion: The imposed social isolation due to the imminent danger of spread appears to have contributed for the aggravation of breast cancer clinical and physical symptoms, such as the perceived increase of lymphedema and pain in the homolateral limb of the surgery, mostly, in addition to causing a significant reduction of physical activity regular practice.

Key words: Exercise; Breast Neoplasms; Coronavirus Infections; Social Isolation.

\section{RESUMEN}

Introducción: El escenario de aislamiento social impuesto por la pandemia Covid-19 ha sido un desafío para la salud física y mental de las mujeres en tratamiento por cáncer de mama. Objetivo: Investigar la percepción de los impactos de la práctica de actividad física a distancia sobre los síntomas clínicos y físicos de mujeres con cáncer de mama, durante el aislamiento social impuesto por la pandemia de Covid-19. Método: Estudio cualitativo y exploratorio con 32 participantes con cáncer de mama, de un programa de extensión en una universidad pública del Sur de Brasil. El guion de la entrevista, aplicado en los meses de abril y mayo de 2020, estuvo compuesto por 30 preguntas abiertas y cerradas, cuyos datos fueron transcritos íntegramente y analizados mediante la técnica de Análisis del Discurso. Resultados: En el análisis se destacaron tres aspectos (categorías a priori), considerando el efecto del tiempo, a saber: a) aspectos personales y clínicos; b) aspectos físicos y clínicos antes y durante el aislamiento social (nivel de actividad física, dolor y disposición general); c) aspectos relacionados con la práctica de actividad física realizada de forma remota (percepciones generales sobre adaptaciones a actividades remotas y dificultades para realizar práctica en el entorno del hogar). Conclusión: El aislamiento social que impone el inminente peligro de contagio parece haber contribuido al agravamiento de los síntomas clínicos y físicos del cáncer de mama, como la percepción de aumento de linfedema y dolor, especialmente en el brazo correspondiente a la cirugía oncológica, además de reflexionar sobre una disminución significativa de la actividad física regular.

Palabras clave: Ejercicio Físico; Neoplasias de la Mama; Infecciones por Coronavirus; Aislamiento Social.

\footnotetext{
1-5 Universidade do Estado de Santa Catarina (UDESC). Centro de Educação Física, Fisioterapia e Desportos (CEFID). Programa de Pós-Graduação em Ciências do Movimento Humano (PPGCMH). Florianópolis (SC), Brasil.

'E-mail: vanessablyra@gmail.com. Orcid iD: https://orcid.org/0000-0001-9653-2930

${ }^{2}$ E-mail: tatibem@hotmail.com. Orcid iD: https://orcid.org/0000-0002-9735-3472

${ }^{3}$ E-mail: francinestein@unidavi.edu.br. Orcid iD: https://orcid.org/0000-0001-6501-0259

${ }^{4}$ E-mail: fabiana.sperandio@udesc.br. Orcid iD: https://orcid.org/0000-0002-2435-0246

${ }^{5}$ E-mail: adriana.guimaraes@udesc.br. Orcid iD: https://orcid.org/0000-0001-5167-2921

Endereço para correspondência: Vanessa Bellani Lyra. Rua Professor João José Cabral, 127 - apto. 201 - Balneário. Florianópolis (SC), Brasil. CEP 88075-535.

E-mail: vanessablyra@gmail.com
} 


\section{INTRODUÇÃO}

Em março de 2020, a Organização Mundial da Saúde (OMS) declarou o surto da doença pelo coronavírus 2019 (coronavirus disease 2019 - Covid-19) como uma pandemia do novo coronavírus, sendo este mortal e de alta contaminação $0^{1,2}$. Durante a pandemia, todos os países adotaram estratégias de distanciamento social e restriçôes com o intuito de reduzir a propagação do vírus; assim, novas medidas de atendimentos foram adotadas, como acolhimentos on-line ou telemedicina ${ }^{3}$.

As tentativas de conter a Covid-19 forçaram os países a aderirem ao isolamento e ao distanciamento social. As mulheres com diagnóstico de câncer de mama são consideradas uma populaçáo vulnerável e apresentam sintomas graves causados pela Covid-194. Entretanto, o distanciamento social e a interrupção das atividades realizadas, como a prática de exercício físico e/ou fisioterapia, podem ter um impacto negativo na saúde global dessas mulheres. Parte do desafio neste momento é continuar a fornecer os cuidados clínicos necessários de uma maneira segura tanto para os profissionais da saúde quanto para essa população.

Sendo assim, a opçáo atual são os atendimentos remotos, por serem seguros, criando-se estratégias para a manutenção da saúde dessas mulheres ${ }^{3}$. A literatura evidencia, por meio de uma metanálise, que a atividade física é essencial a essa população e pode reduzir a recorrência do câncer de mama e ainda minimizar os riscos de todas as causas de morte provocados pela doença $a^{5}$; além disso, a Sociedade Americana de Câncer recomenda a atividade física para a prevenção do câncer ${ }^{6}$; ou seja, em um momento em que não é possível ter-se atividade física presencial, novas estratégias são necessárias, como, por exemplo, aulas remotas diárias, uma vez que ficar sem a atividade física não é uma possibilidade. Sendo assim, o objetivo deste estudo é investigar a prática de atividade física remota e suas implicaçóes durante isolamento social imposto pela pandemia da Covid-19 em mulheres com câncer de mama.

\section{MÉTODO}

Estudo qualitativo e exploratório na área da saúde ${ }^{7-10}$, aprovado pelo Comitê de Ética em Pesquisa em Seres Humanos (CEPSH) da Universidade do Estado de Santa Catarina (UDESC), sob o número de protocolo 3.985.052. Todos os procedimentos seguiram a declaração de Helsinki.

Foram alocadas para o estudo as participantes de um programa de extensão (Ritmo e Movimento) de uma universidade pública do Sul do Brasil, que atenderam aos critérios de inclusão: faixa etária maior do que 18 anos; estádio clínico I a III do câncer de mama; e que estavam recebendo atividade física de forma remota (vídeos on-line e lives) e informaçóes sobre atividades físicas por meio de aplicativos de comunicação digital e/ou redes sociais, durante o isolamento social em função da pandemia da Covid-19. Como critérios de exclusão: não estar recebendo atividades físicas de forma remota. Dessa forma, das 32 participantes do programa, cinco não puderam participar do estudo; quatro por não atenderem às ligaçôes e uma pela impossibilidade de conclusão da entrevista por razóes pessoais, totalizando 22 participantes $(58,23 \pm 6,4$ anos) que realizaram a entrevista.

Como técnica de coleta de dados, fez-se uso da entrevista qualitativa, organizada de forma semiestruturada ${ }^{8,11-13} \mathrm{e}$ individual ${ }^{14}$. O roteiro de entrevista continha 30 questóes organizadas em estruturas abertas e fechadas, subdivididas em três blocos e suas respectivas categorias a priori: a) aspectos pessoais e clínicos (escolaridade, ocupação, estado conjugal, aspectos cirúrgicos, recidiva, tratamento adjuvante de hormonioterapia, quimioterapia prévia e radioterapia prévia); b) aspectos físicos antes e durante o isolamento social (nível de atividade física, dor e disposição geral); e c) aspectos relacionados à prática de atividade física realizada de forma remota (percepçôes gerais acerca das adaptaçôes às atividades remotas e dificuldades em realizar a prática no ambiente domiciliar).

Por sua vez, as categorias citadas permitiram o agrupamento e a organização das ideias trazidas pelas falas, as quais foram interpretadas a partir da técnica de Análise de Discurso ${ }^{15}$. Ao utilizar-se dessa técnica, é importante destacar que procura-se entender não só a palavra, mas o contexto histórico-social e o espaço do tempo em que ocorre o discurso e seus efeitos/produção de sentidos.

As entrevistas foram conduzidas por duas pesquisadoras (VBL e TBF) do Laboratório de Pesquisa em Lazer e Atividade Física (LAPLAF/CNPq), por meio de chamada telefônica, cujas tentativas de realização das chamadas chegavam ao número de cinco, sendo desconsiderada a participação, caso a chamada não fosse atendida nesse intervalo de tempo.

A duração média das entrevistas foi de 25 minutos e as falas foram registradas pelo software Gravador de Chamadas versão 2.3.7, cuja utilização permitiu a gravação automática das chamadas telefônicas. A transcriçáo das entrevistas foi realizada por uma terceira pesquisadora (FS), colaboradora também do LAPLAF, por meio do aplicativo Speechnotes - versão 1.7.0, sendo a coleta de dados realizada nos meses de abril e maio de 2020 . Cabe destacar que, quando da transcriçāo, as falas das participantes foram ajustadas para a correta ortografia da língua portuguesa, com vistas a facilitar o seu 
entendimento e evitar constrangimentos, observando o cuidado ético de preservar o sentido original do discurso.

\section{RESULTADOS}

As respostas obtidas por meio das entrevistas permitiram construir um breve panorama das principais adaptaçôes físicas e clínicas percebidas em si pelas participantes, a partir da prática de atividade física remota. Três categorias a priori foram inicialmente construídas como organizadoras das informaçōes as quais se buscavam, a saber: a) aspectos pessoais e clínicos; b) aspectos físicos e clínicos antes e durante o isolamento social; c) aspectos relacionados à prática de atividade física realizada de forma remota. $\mathrm{O}$ conhecimento sobre as condiçôes físicas e clínicas do grupo de participantes e sua relação com a situaçâo atual de isolamento social foram objeto de interesse deste estudo e puderam ser reunidos nas seguintes subcategorias $a$ posteriori: nível de atividade física, dor e disposição geral. Do mesmo modo, a compreensão sobre o consumo de atividade física on-line relacionado a tais condiçôes, no período que decorre a pandemia, permitiu a organização nas seguintes subcategorias adiante: percepçóes gerais acerca das adaptaçôes às atividades remotas; e dificuldades em realizar a prática no ambiente domiciliar. Vale ressaltar que 20 mulheres do grupo entrevistado aderiram à prática do isolamento social em suas rotinas diárias, sendo que apenas duas delas continuaram a frequentar, regularmente, seus postos de trabalho.

Diante do cenário de mudanças estruturais impostas às suas rotinas pela pandemia da Covid-19, as participantes foram estimuladas a refletir e a comparar as percepçóes acerca de aspectos de sua saúde física e da rotina de atividade física, nos períodos antes e durante a pandemia.

Entre as 22 participantes do estudo, $41 \%$ tinham ensino médio, $73 \%$, vínculo empregatício, $50 \%$ tinham companheiro, $86 \%$ apresentaram câncer em uma mama, $64 \%$ não realizaram a reconstrução mamária, 54\% estavam em tratamento adjuvante de hormonioterapia, $86 \%$ realizaram quimioterapia prévia, $82 \%$, radioterapia prévia e $100 \%$ aderiram ao isolamento social.

No que diz respeito à disposição geral das participantes, o isolamento social parece ter interferido de forma importante sobre a sensação de cansaço para a realização das atividades de vida diária. No período anterior à pandemia da Covid-19, sensaçóes físicas relacionadas a cansaço, apatia e indisposição praticamente inexistiam nas falas das participantes. De modo particular, três participantes mencionaram já se perceberem diariamente cansadas em suas rotinas desde o diagnóstico de câncer de mama e justificavam tal condição ao efeito colateral do tratamento medicamentoso ao qual estavam submetidas.
Embora a nova rotina tenha imposto às dinâmicas práticas um ritmo menos acelerado, é justamente nessa falta de fluidez do tempo que a percepção de (im)potência atravessa a fala das participantes. Em outras palavras, não há, propriamente, uma sensação de cansaço físico provocado por esforço ou atividade, mas sim uma "indisposição" causada, justamente, pela impossibilidade de esforçar-se fisicamente. Como resultado, algumas participantes mencionaram que pequenas açôes diárias se tornaram mais exigentes às qualidades físicas, outrora recrutadas com grande facilidade, como, por exemplo, tomar banho e secar os cabelos.

Com o isolamento social, as dores corporais parecem ter aumentado em expressão. Muitas participantes mencionaram que o convívio com as dores físicas, em diversas partes do corpo, já era uma situação habitual em suas rotinas, no período pré-pandemia, e que estas permaneceram no período atual, porém, algumas delas, em maior intensidade. Foram mencionadas dores nas pernas, nos ossos, nas costas, no nervo ciático e articulaçôes em geral, ao passo que a dor no braço correspondente ao seio em que fora realizada a cirurgia de retirada do tumor se destacou entre todas as dores percebidas. Do grupo de participantes, nove mencionaram a dor causada pelo "inchaço" do braço (linfedema) como aquela mais persistente, incômoda e crescente durante o período de isolamento. Algumas das participantes, como foi o caso da participante 21 , ainda associaram o referido inchaço ao fato de estarem convivendo em um ambiente com mais tensão emocional:

Dor eu sentia e ainda sinto! Muitas dores nos braços e nos ombros. Eu acho que pode ser sim [efeito da tensão emocional] porque toda vez que eu vou no médico e reclamo disso, ele fala que é tensão, que eu sou muito tensa. Essa semana, mesmo, eu fui ao médico cardiologista porque, segundo ele, eu estou com o coraçáo meio acelerado. [Sempre fui ansiosa, mas] não tanto como depois que eu fiquei em isolamento. Desde quando eu fiz a minha cirurgia e o tratamento de câncer, eu fiquei muito medrosa, eu tenho muito medo, muito medo mesmo! [...] Eu sinto bastante dor nos dois braços e costas, eu durmo, acordo, durmo e acordo e a dor permanece! Um dia menos, outro dia um pouquinho mais e, assim, vai indo. Hoje eu não estou com muita dor, mas teve dias que eu já tive muita dor (Participante 21).

Ainda nesse espectro, cinco participantes associaram o aumento da dor percebida no braço à diminuição brusca de movimentação corporal, imposta pela nova rotina, no isolamento. $\mathrm{O}$ fato de permanecer em casa deslocando-se pouco, não estar vinculada aos afazeres 
de trabalho ou círculo social ou, ainda, não ter o corpo submetido a esforços físicos maiores, aparece, na fala dessas participantes, como elementos centrais no surgimento de dores e inchaços crescentes nos braços:

[Agora] a gente fica muito parada, e o fato da gente se movimentar não forma tanto líquido no braço! O movimento é importante porque a gente já quase perdeu o movimento do braço e do ombro. As costas e a lateral direita me incomodam muito (Participante 12).

De outro modo, os relatos ainda permitiram perceber que a dor física foi acompanhada de perto pela dor emocional, ainda que de forma indireta. Tal relaçáo foi claramente verbalizada na fala da participante 2, para quem o isolamento social trouxe dores emocionais mais profundas e dolorosas do que, propriamente, as físicas: "Não poder se ver, se abraçar, isso é uma grande dor".

Apenas quatro mulheres pontuaram não sentirem dores físicas, seja no período anterior ou durante a pandemia, e ainda algumas delas utilizaram o sinônimo "desconfortos" ao se referirem propriamente às percepçóes de suas dores. Vale ressaltar que dez participantes pontuaram não perceber piora nos sintomas físicos relacionados ao câncer de mama.

No que tange ao nível de atividade física, um número instigante é capaz de expressar os reflexos trazidos pelo isolamento social, que pontua o crescimento das participantes que se percebem sedentárias. Se antes apenas três delas mencionaram exercer em suas rotinas o comportamento sedentário, em relação à prática de atividade física, no período que segue, esse número passa a ser de 12; ou seja, atinge mais da metade do grupo analisado. As razóes para a adoção do comportamento sedentário, em meio à pandemia, são diversas: falta de motivaçáo; não prioridade dada ao exercício físico na nova rotina; o fato de estar sozinha em casa, sem as companheiras de grupo; a não adaptação ao meio digital ou mesmo a não aceitação das aulas de atividade física pelas estratégias de ensino remoto; e o querer estar com as amigas presencialmente nas atividades práticas. Somadas a tudo isso estão as questóes de ordem prática envolvendo o acesso dificultado à rede de Internet. $\mathrm{O}$ náo saber acessar e a falta de um local apropriado no ambiente das residências para as referidas práticas parecem ter acentuado o aumento significativo no número de participantes que se tornaram sedentárias:

Hoje [minha condição de saúde é] sedentária. Eu acordo às 11 horas quando dá vontade. Vou dormir a uma hora ou duas horas [da manhá] e com esse friozinho é que eu fico na cama, mesmo! Eu estou recebendo [as atividades físicas on-line], mas não está pegando direito aqui o meu sinal de Internet. Para receber, eu tenho que ir lá fora, e aqui eu também não tenho onde fazer exercício, a minha casa é muito pequenininha. [...] daí eu tenho que abrir meus dados móveis e gasta muito porque eu tenho limite e eu tenho que economizar (Participante 5).

De um modo geral, os impactos ocorridos com relação aos aspectos físicos, até então apresentados, estão intimamente relacionados à prática regular de atividades físicas ou à falta/diminuição dela. Com a deflagração da pandemia da Covid-19 e o consequente período de isolamento social imposto pelas autoridades de saúde internacionais e locais, as rotinas de atividades físicas do grupo de participantes precisaram ser reorganizadas e adaptadas para o ensino remoto, por meio de vídeos, lives e/ou encontros síncronos. Todas se encontravam com a teleorientação profissional e recebendo vídeos e instruçôes para realização da prática.

Os tipos mais praticados de atividade física por elas antes da pandemia eram caminhada, musculação, dança, alongamento, ginástica e treinamento funcional. Dez possuíam frequência de atividade física de duas vezes na semana e oito com frequência semanal superior, sendo de três a sete vezes na semana. Durante o isolamento social, as principais atividades físicas praticadas concentraram-se em caminhadas, dança, pilates e alongamento. De outro modo, com o isolamento social, a frequência não manteve a assiduidade.

As principais adaptaçôes, que foram necessárias para a prática de atividade física on-line, tangenciaram questôes de ordem operacional, relacionadas ao ajuste dos ambientes das casas e acesso à Internet e, também, questôes subjetivas, relacionadas à necessidade de ressignificação da própria atividade física, seus conceitos e finalidades. Desse modo, para além da participante 18, outras 14 descreveram não apreciar ou não conseguir acompanhar as atividades físicas on-line:

Não gosto! Tanto que eu faço aula de canto, começamos este ano, marcamos aula de canto, aí depois veio a pandemia, aí a professora disse 'ah vamos fazer aula on-line', eu não quis, eu preciso, eu necessito da presença da pessoa junto comigo, eu acho muito frio sem (Participante 18).

O novo formato de aulas trouxe consigo a falta do ambiente de grupo para a prática de atividade física. A presença das colegas, o ambiente harmonioso e descontraído, gerado a partir dos encontros semanais, parecem ser elementos estruturais importantes para a manutenção do hábito e da motivação para a realização das 
atividades. Assim, os principais pontos mencionados como dificuldades a partir da prática de atividade física on-line estão relacionados às questóes de manter a regularidade da prática sozinhas, a falta de motivação e, propriamente, o sentimento de solidão:

É o fato de estar sozinha mesmo, estar realizando aula sozinha, a gente acaba ficando com preguiça, a troca com outro é muito bom, mesmo às vezes quando você não está com vontade, você chega lá, conversa e acaba se animando (Participante 12).

Importa destacar que $100 \%$ das participantes mencionaram preferir realizar atividades físicas de forma presencial ao formato on-line.

Para além dessas questóes, pontuou-se, também, que a incerteza em executar os exercícios de forma correta também se configurava uma grande dificuldade para esse momento. Diante desse fato, parece ter ficado comprometida a aprendizagem de novos movimentos, fazendo com que a prática atual se concentrasse em movimentos já previamente vivenciados e aprendidos. Cabe ressaltar que, mesmo diante da não adesão ou não apreciação, as participantes consideravam válidas e importantes as práticas on-line, afirmando que as indicariam a outras pessoas na mesma situação de saúde.

\section{DISCUSSÃO}

Constatou-se que o isolamento social parece ter contribuído para o acirramento dos sintomas clínicos e físicos do câncer de mama, tais como o aumento percebido do linfedema e as dores, sobretudo no braço correspondente à cirurgia oncológica, além de refletir em uma diminuição significativa da prática regular de atividade física. Em relação à prática de atividades físicas remotas, deflagra-se um cenário de grandes dificuldades de ordem prática e conceitual, bem como apresenta-se como uma estratégia que desperta um baixo interesse às participantes.

As mulheres com câncer de mama na era Covid-19 têm um maior risco de contrair a doença, assim como um pior resultado clínico em comparação com a população em geral $^{4}$. Em virtude dos problemas mencionados, o lockdown, o distanciamento social e o isolamento social foram necessários por causa do aumento de casos da Covid-19. Essas participantes deixaram de pertencer ao grupo de atividade física para mulheres com câncer de mama em consequência dessa nova rotina, bem como, por medo de contrair a doença, adquiriram um comportamento sedentário. Destaca-se que a cidade de Florianópolis - SC entrou em período oficial de "quarentena" na data de 16 de março de 2020, de acordo com decreto oficial ${ }^{16}$ expedido pelas autoridades competentes. Desde o início da pandemia, a cidade já havia contabilizado, até agosto, 5.335 casos confirmados e 111 óbitos $^{17}$.

Diante dessa situação de crise global, novas estratégias foram adotadas, a fim de oferecer continuidade às atividades com segurança aos profissionais e às participantes, como as atividades remotas, e de minimizar os efeitos colaterais causados pela inatividade física; porém, a preferência delas era pelas atividades presenciais, em razão do laço afetivo e do acolhimento concebido durante as atividades presenciais, por se tratar de um momento de troca de experiências no qual elas sentiam-se à vontade para falar sobre o câncer. Nesse período importante na vida dessas mulheres, de expressarem as suas dores físicas e emocionais, essa humanização constituiu um vínculo entre os profissionais e as participantes.

As intervenções para essas participantes são essenciais para a prevenção e o tratamento das complicaçóes pós-operatórias precoces e tardias, os atendimentos remotos podem ajudar de modo geral a saúde e, ao mesmo tempo, a melhorar a qualidade de vida. Estudo realizado por Meneses et al. ${ }^{18}$ com Telehealth verificou que o estado emocional e o bem-estar físico permaneceram o mesmo, porém os níveis de dor melhoraram durante o período de intervenção.

Evidencia-se, por meio de revisão sistemática, que a atividade física melhora as dores de mulheres com câncer de mama $^{19}$. A dor é uma variável multifatorial e fatores emocionais podem influenciar na sua interpretação, ela é crescente em decorrência dos novos hábitos de vida como o sedentarismo ${ }^{20}$. Entre as participantes, foi relatada dor no membro homolateral à cirurgia e na mama. A dor, como as comorbidades em geral, é considerada um tipo específico de sofrimento e o exercício físico é capaz de promover melhora dos sintomas referentes à dor ${ }^{20,21}$. Uma vez que a maioria dessas participantes estava sem fisioterapia e prática de atividade física, cogita-se o aumento das dores, implicando de forma negativa no dia a dia.

Este estudo apresentou limitaçôes. Algumas das participantes podem ter respondido às perguntas com certo grau de incompreensão por se tratar de uma pesquisa feita apenas por meio telefônico, porém, buscou-se deixar as perguntas claras e diretas. A pequena amostra de participantes foi recrutada em um grupo de mulheres em tratamento do câncer de mama, frequentadoras de um programa acadêmico de extensão universitária, e pode não ter sido representativa de mulheres com câncer de mama em outras Regiōes Geográficas do Brasil. Portanto, os resultados deste estudo não podem ser generalizados para outras mulheres com câncer de mama. 


\section{CONCLUSÃO}

O isolamento social imposto pelo perigo iminente do contágio, sobretudo a pessoas pertencentes a grupos de risco, como mulheres com câncer de mama, parece ter contribuído para o acirramento das dores físicas e emocionais; o aumento da sensação de cansaço para a realização das atividades de vida diária; e um crescimento significativo das participantes que assumiram o comportamento sedentário. $\mathrm{O}$ novo ambiente de reservas e limitaçóes de movimento, conduzido pela prática de atividade física de forma on-line, parece não favorecer um estilo de vida ativo, na medida em que dificuldades de ordem prática - como acesso à Internet ou espaço para a realização de atividades - e conceituais - como a pouco aderência às novas estratégias remotas, a falta de motivação e a ausência dos pares - se apresentam diante das novas rotinas impostas às participantes. Desse modo, torna-se importante a atenção dada a realizaçáo de atividades físicas e seus impactos na saúde integral de participantes em tratamento do câncer de mama, durante a pandemia da Covid-19, a fim de que se possam minimizar os impactos negativos causados pelo isolamento social.

\section{CONTRIBUIÇÕES}

Todas as autoras contribuíram na concepção el ou no planejamento do estudo; na obtençáo, análise e interpretação dos dados; assim como na redação e revisão crítica; e aprovaram a versão final a ser publicada.

\section{DECLARAÇÃO DE CONFLITO DE INTERESSES}

Nada a declarar.

\section{FONTES DE FINANCIAMENTO}

Não há.

\section{REFERÊNCIAS}

1. Baloch S, Baloch MA, Zheng T, et al. The coronavirus disease 2019 (COVID-19) pandemic. Tohoku J Exp Med. 2020;250(4):271-8. doi: https://doi.org/10.1620/ tjem.250.271

2. Kakodkar P, Kaka N, Baig MN. A comprehensive literature review on the clinical presentation, and management of the pandemic coronavirus disease 2019 (COVID-19). Cureus. 2020;12(4):e7560. doi: https:// doi.org/10.7759/cureus. 7560

3. Viale G, Licata L, Sica L, et al. Personalized riskbenefit ratio adaptation of breast cancer care at the epicenter of Covid-19 outbreak. Oncologist. 2020;25(7):e1013-e1020. doi: https://doi.org/10.1634/ theoncologist.2020-0316

4. Soran A, Gimbel M, Diego E. Breast cancer diagnosis, treatment and follow-up during COVID-19 pandemic. Eur J Breast Health. 2020;16(2):86-88. doi: https://doi. org/10.5152/ejbh.2020.240320

5. Spei ME, Samoli E, Bravi F, et al. Physical activity in breast cancer survivors: a systematic review and metaanalysis on overall and breast cancer survival. Breast. 2019;44:144-52. doi: https://doi.org/10.1016/j. breast.2019.02.001

6. Rock CL, Thomson C, Gansler T, et al. American Cancer Society guideline for diet and physical activity for cancer prevention. CA Cancer J Clin. 2020;70(4):245-71. doi: https://doi.org/10.3322/caac.21591

7. Hudelson PM. Qualitative research for health programs. Geneva: WHO; 1994.

8. Minayo MCS. O desafio do conhecimento: pesquisa qualitativa em saúde. 9. ed. São Paulo: Hucitec; 2006.

9. Becker HS. Método de pesquisa em ciências sociais. São Paulo: Hucitec; 1993.

10. Yin RK. Estudo de caso: planejamento e métodos. 3. ed. Porto Alegre: Bookman; 2005.

11. Duarte J, Barros A, organizadores. Métodos e técnicas de pesquisa em comunicação. 2. ed. São Paulo: Atlas; 2006.

12. Sade C, Barros LRM, Melo JJM. O uso da entrevista na pesquisa-intervenção participativa em saúde mental: o dispositivo GAM como entrevista coletiva. Ciênc Saúde Coletiva. 2020;18(10):2813-24. doi: https://doi. org/10.1590/S1413-81232013001000006

13. Fontanella BJB, Campos CJG, Turato ER. Coleta de dados na pesquisa clínico-qualitativa: uso de entrevistas não-dirigidas de questóes abertas por profissionais da saúde. Rev Lat-Am Enf [Internet]. 2006 [acesso 2020 ago 26];14(5). Disponível em: https://www.scielo.br/ pdf/rlae/v14n5/pt_v14n5a25.pdf

14. Bauer MW, Gaskell G, organizadores. Pesquisa qualitativa com texto, imagem e som: um manual prático. Petrópolis: Vozes; 2002. Capítulo 3, Gaskell G. Entrevistas individuais e grupais; p. 64-89.

15. Orlandi EP, Lagazzi-Rodrigues $S$, organizadores. Introdução às ciências da linguagem: discurso e textualidade. São Paulo: Pontes; 2006.

16. Santa Catarina (Estado). Decreto no 506, de 12 de março de 2020. Dispóe sobre as medidas para enfrentamento da emergência de saúde pública de importância internacional decorrente do coronavírus e estabelece outras providências [Internet]. Diário Oficial do Estado de Santa Catarina, 2020 mar. 12, n. 21.220 , p. 5 [acesso 2020 ago 26]. Disponível em: https://leisestaduais.com. br

17. Plataforma BoaVista [Internet]. Florianópolis, SC: Centro de Informática e Automação do Estado de Santa 
Catarina [data desconhecida]. [acesso 2020 ago 26]. Disponível em: https://www.sc.gov.br/boavista

18. Meneses K, Gisiger-Camata S, Benz R, et al. Telehealth intervention for Latina breast cancer survivors: a pilot. Womens Health (Lond). 2018;14:1745506518778721. doi: https://doi.org/10.1177/1745506518778721

19. Fretta TB, Boing L, Bussmann RM, et al. Tratamento de reabilitação para dor em mulheres com câncer de mama. BrJP. 2019;2(3):279-83. doi: https://doi. org/10.5935/2595-0118.20190049

20. Kopec JA, et al. Relationship between arm morbidity and patient-reported outcomes following surgery in women with node-negative breast cancer: NSABP protocol B-32. J Support Oncol. 2013;11(1):22-30. doi: https://doi. org/10.1016/j.suponc.2012.05.003

21. Sousa E, Carvalho FN, Bergmann A, et al. Funcionalidade de membro superior em mulheres submetidas ao tratamento do câncer de mama. Rev Bras Cancerol. 2013;59(3):409-17. doi: https://doi.org/10.32635/21769745.RBC.2013v59n3.506 